\title{
Incidence and Prevalence of Overt Hypothyroidism and Causative Diseases in Korea as Determined Using Claims Data Provided by the Health Insurance Review and Assessment Service
}

\author{
Young Nam Kim¹, Gi Hyeon Seo², Yoon Young Cho', Hye-In Kim¹, Hosu Kim¹, Tae Hyuk Kim¹, Jae Hoon Chung', Sun Wook Kim \\ ${ }^{1}$ Division of Endocrinology and Metabolism, Department of Medicine, Samsung Medical Center, \\ Sungkyunkwan University School of Medicine, \\ ${ }^{2}$ Health Insurance Review and Assessment Service, Seoul, Republic of Korea
}

\section{BACKGROUND}

A prospective cohort study of the community population is required to determine the incidence and prevalence of hypothyroidism. However, such large-scale epidemiological studies face time and cost constraints.

Therefore, investigations of incidence and prevalence are mainly conducted in limited community cohorts including middle-aged women, elderly patients, health examinees visiting clinics, or patients admitted to hospital. It is difficult to determine the exact incidence and prevalence of overt hypothyroidism because they differ according to the screening test for hypothyroidism or the study subjects.

From reports from foreign countries, the prevalence of overt hypothyroidism that was not previously diagnosed is $0.2 \%$ to $0.4 \%$, and the rate is about $1 \%$ to $2 \%$ when previously diagnosed cases and cases that develop after thyroidectomy or radioactive iodine (RAI) treatment are included. But there has been no nationwide survey to obtain data in Korea

\section{OBJECTIVE}

We investigated the incidence and prevalence of hypothyroidism in patients who have been prescribed thyroid hormone using claims data provided by the Health Insurance Review and Assessment Service (HIRA) The incidence and prevalence of hypothyroidism according to the causative diseases were also evaluated.

\section{METHODS}

In this study, claims data for the prescription of thyroid hormone (levothyroxine) from HIRA for 2008 to 2012 were evaluated. The principal diagnosis and first subdiagnosis in the year before and year of thyroid hormone prescription were analyzed to evaluate the causative diseases that were treated with thyroid hormone. Hypothyroidism was defined in this study as overt hypothyroidism with long-term prescription of thyroid hormone.

\section{RESULTS}

A total of 541,969 hypothyroidism patients $(92,832$ men and 449,137 women) were prescribed thyroid hormone between 2008 and 2012. Their mean age was $48.7 \pm 14.8$ years. The annual incidence in 2008 to 2012 was 2.26 per 1,000 population ( 0.78 in men and 3.72 in women).

\section{Age and gender specific-incidence of overt hypothyroidism in Korea}

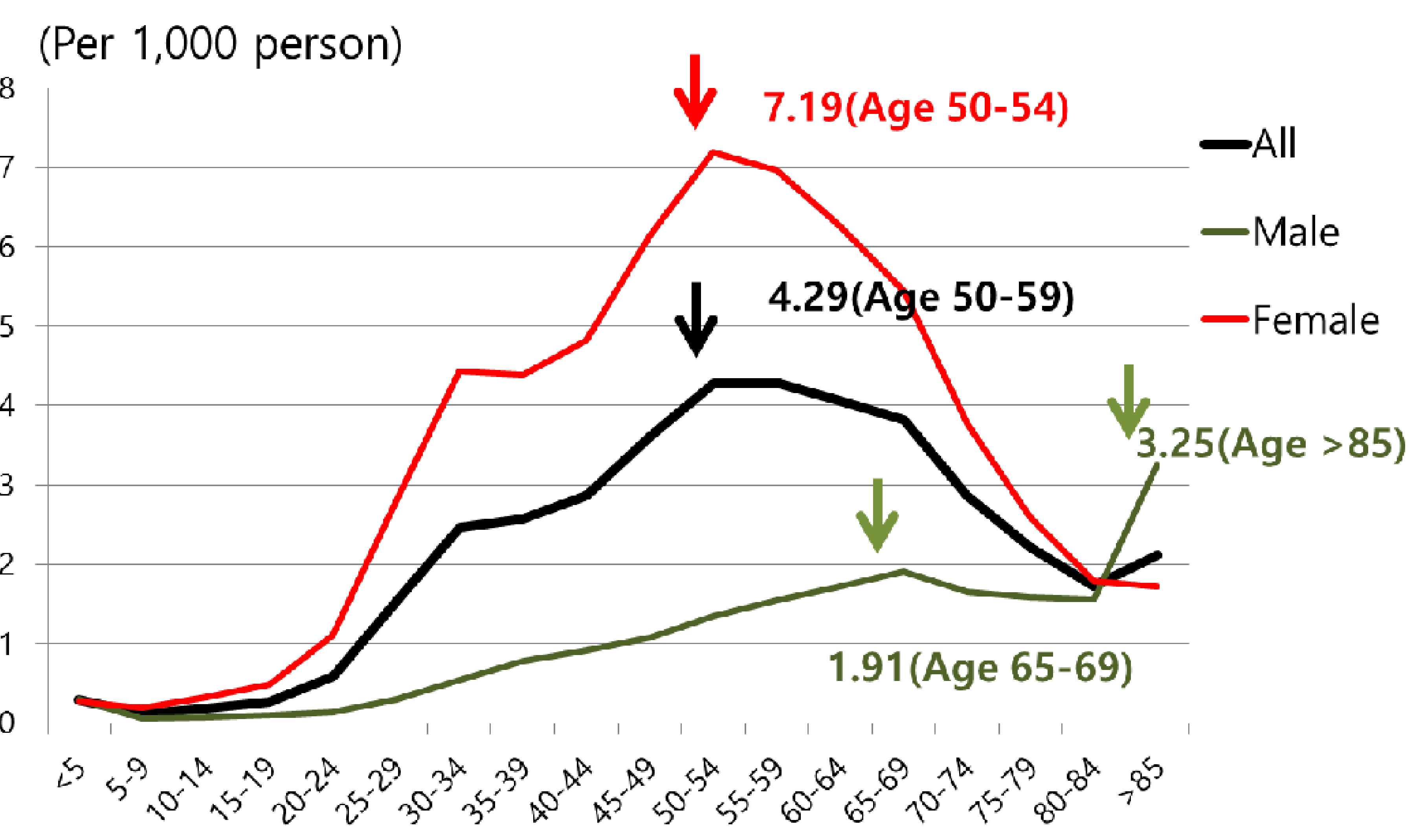

It increased with age in men, with two peaks: a minor peak at 65 to 69 years $(1.91$ per 1,000$)$ and a major peak at $>85$ years $(3.25$ per 1,000$)$. It also increased with age in women, with a peak at 50 to 54 years $(7.19$ per $1,000)$
Causative disease of overt hypothyroidism in Korea (2008-2012)
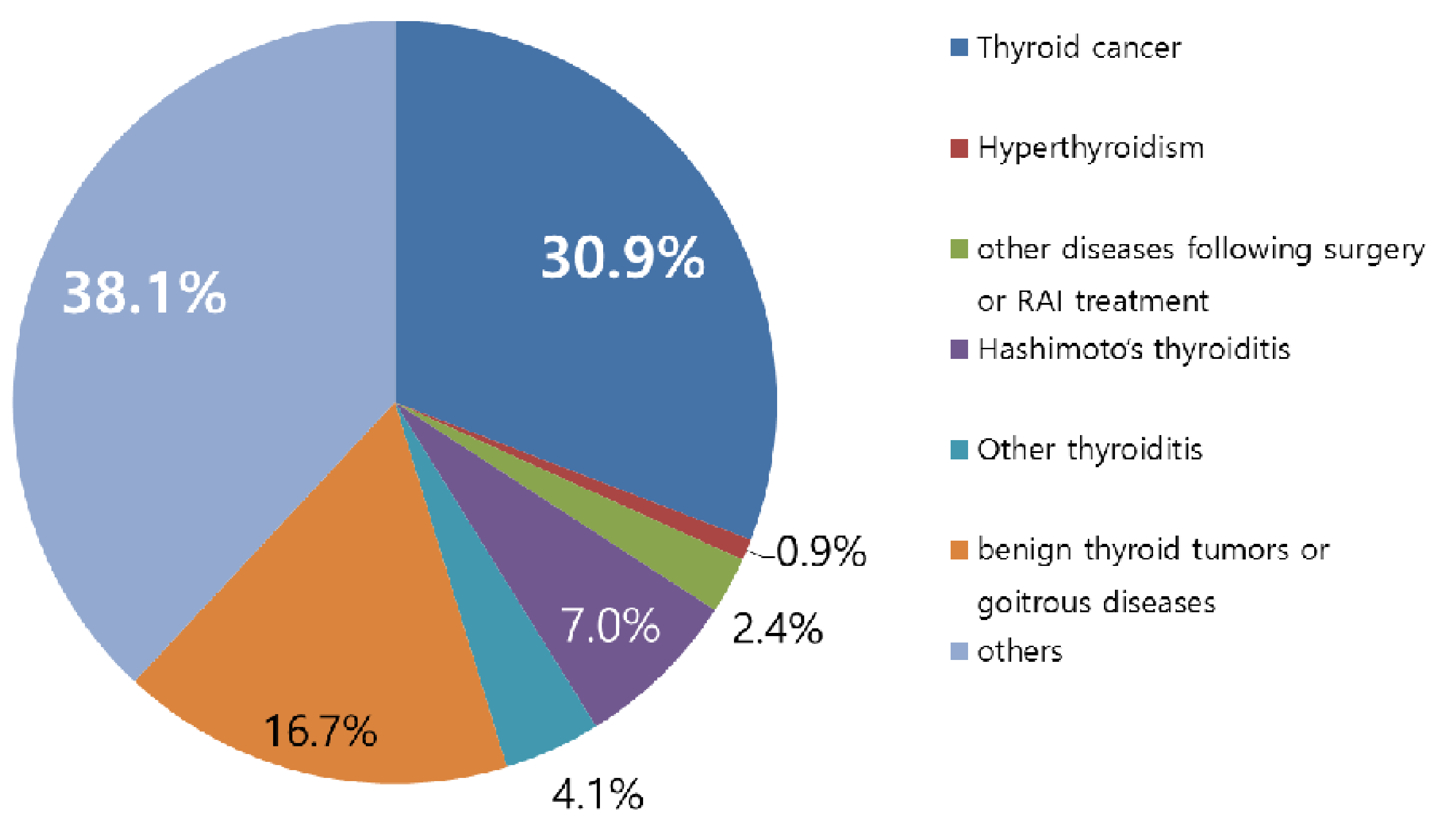

We classified 541,969 hypothyroidism patients prescribed thyroid hormone between 2008 and 2012 into seven categories according to the causative disease: (1) 167,335 patients (30.9\%) with thyroid cancer; (2) 4,811 patients $(0.9 \%)$ with hyperthyroidism; (3) 12,775 patients $(2.4 \%)$ with diseases other than thyroid cancer and hyperthyroidism following surgery or RAI treatment; (4) 37,841 patients (7.0\%) with Hashimoto's thyroiditis; (5) 22,175 patients (4.1\%) with other thyroiditis; (6) 90,688 patients $(16.7 \%)$ with benign thyroid tumors or goitrous diseases; and (7) 206,344 patients $(38.1 \%)$ with diseases with prescription of thyroid hormone not covered by criteria (1) to (6).

\section{Annual incidence of causative disease for overt hypothyroidism in Korea (2008-2012)}

\begin{tabular}{|l|c|c|}
\hline \multicolumn{1}{|c|}{ Category } & $\begin{array}{c}\text { Incidence } \\
\text { (per 1,000 person) }\end{array}$ & Peak age \\
\hline Thyroid cancer & 0.70 & $50 \mathrm{~s}$ \\
\hline Hyperthyroidism & 0.02 & \\
\hline
\end{tabular}

Other than cancer/hyperthyroidism

0.05

Annual incidence
except for cancer:
$1.56 / 1,000(0.2 \%)$

Hashimoto's thyroiditis

0.16

Male 60s, Female early 30 s, 50 s

Other thyroiditis

0.09

Benign tumor/goiter

0.38

Male 60 s, female 50 s

Others

0.86

The annual incidence per 1,000 population was $0.70,0.02,0.05,0.16,0.09$, 0.38 , and 0.86 for thyroid cancer, hyperthyroidism, other diseases following surgery or RAI treatment, Hashimoto's thyroiditis, other thyroiditis, benign thyroid tumors or goitrous diseases, and diseases with prescription of thyroid hormone not covered by criteria (1) to (6), respectively. When 167,335 patients with thyroid cancer were excluded, there were 374,634 hypothyroidism patients, and the annual incidence of hyperthyroidism was 1.56 per 1,000 population (1.68 in 2008, 1.52 in 2009, 1.61 in 2010, 1.58 in 2011, and 1.41 in 2012).

For thyroid cancer, the incidence was highest in the $50 \mathrm{~s}$ in both men and women; for Hashimoto's thyroiditis and other thyroiditis, the incidence was highest in the $60 \mathrm{~s}$ in men and the early $30 \mathrm{~s}$ and early $50 \mathrm{~s}$ in women. For benign thyroid tumors or goitrous diseases, the incidence was highest in the $60 \mathrm{~s}$ in men and the $50 \mathrm{~s}$ in women.

\section{CONCLUSION}

This is a report of the first nationwide investigation of the incidence and prevalence of overt hypothyroidism in Korea, although it is limited to patients treated with thyroid hormone.

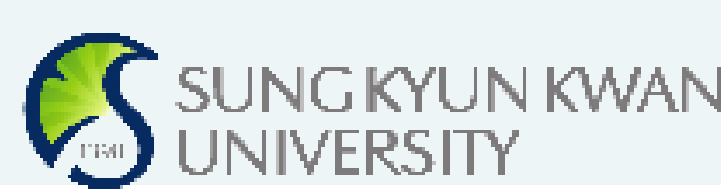

SAMSUNG MEDICAL CENTER 